\title{
ON THE DIMENSION OF THE BOUNDARY OF CLUMPS IN A MULTI-TYPE BOOLEAN MODEL
}

\author{
By Peter Hall ${ }^{1}$ AND Jörg Polzehl \\ Australian National University
}

\begin{abstract}
In a fascinating article on models for particulate microstructure, Hermann and Ohser argue that the superposition of stochastically independent Boolean models may be thought of as producing sets whose boundaries have unusual properties of dimension. Hermann and Ohser employ such superposition models to analyse the particulate structure of rust. In the present paper we provide a theoretical foundation for their work, with respect to both the definition of dimension and its statistical estimation. We argue that the dimension of set boundaries is not well defined in either the Minkowski or capacity senses. It can, however, be properly defined using a mathematical formalization of the practical notion of analysing a spatial pattern at different resolution levels. Adopting this as a basis, we consider properties of estimators of dimension.
\end{abstract}

1. Introduction. The dimension of a curve or a surface is a very convenient way of characterizing its roughness. It is canonical, in at least the sense that it is scale-invariant. In this respect it is unlike other commonly used descriptors of irregularity. Its application to engineering problems involving curves is well known; see, for example, Berry and Hannay (1978), Mandelbrot, Passoja and Paullay (1984), Carter, Cawley and Mauldin (1988), Thomas and Thomas (1988), Dubuc, Zucker, Tricot, Quiniou and Wehbi (1989) and Ling (1990). There the random curves under analysis are typically obtained by drawing a stylus or optical profilometer across a surface, and are often modelled as functions of Gaussian processes.

Hermann and Ohser (1993) also address irregular curves arising in problems of engineering origin, but depart from tradition by treating genuinely two-dimensional patterns and employing intrinsically geometric models. The statistical problem that Hermann and Ohser discuss is motivated by a need to develop models for finely particulated processes in the plane, such as appear in a section through clumps of oxidized material. Clumps are produced by an aggregation of overlapping particles whose centers have been laid down independently and at random. This strongly motivates the use of Boolean models. There particle centers may be treated as points of independent marked Poisson processes in the plane, and the particles themselves are

Received April 1994; revised August 1995.

${ }^{1}$ Also affiliated with CSIRO, Division of Mathematics and Statistics, Sydney, Australia.

AMS 1991 subject classifications. Primary 62H11, 62M30; secondary 62M40.

Key words and phrases. Poisson processes, roughness, scaling model, self-similarity, spatial pattern. 
random shapes whose configurations, like those of the actual particles, are independently distributed, with scales depending on the marks of the point processes.

Unlike the usual Boolean model, where the sizes of most particles are within a relatively narrow band, the data analysed by Hermann and Ohser demand an extremely broad distribution of particle size. The model that they develop produces a spatially homogeneous pattern where the number of particles within any given region is infinite with probability 1 , yet the expected proportion of the region covered by particles is strictly less than 1 . This is achieved by having a sequence of independent marked Poisson processes in the plane, with geometrically increasing intensities, and centering at those points a sequence of random sets with geometrically decreasing sizes. Appropriate choice of set diameters and point process intensities produces the properties noted above. As Hermann and Ohser point out, there is a sense in which the relationship between particle scale and point process intensity may be ascribed a "dimension" which characterizes the critical features of the model.

In the present paper we develop the concept of multitype Boolean models, where the total number of set centers in each nondegenerate region is infinite with probability 1 . The notion of dimension is investigated in that context. It may be shown that the Minkowski dimension of the total boundary of the Boolean model within any finite region is not well defined, except as the value 2. This is related to the fact that Minkowski dimension is not countably stable [e.g., Falconer (1990)]. There are similar problems in defining capacity dimension. Nevertheless, the geometric relationship between the sizes of random sets and point process intensity, at all levels of a scaling model, clearly has features of self-similarity. Motivated by this property and by Hermann and Ohser's practical suggestion that the coverage pattern be analysed at varying levels of resolution, we suggest a concise definition of dimension. A rigorous mathematical argument shows that the definition is meaningful for a wide range of multitype Boolean models, including those employed by Hermann and Ohser. We discuss estimation of dimension.

Our theoretical development of multitype Boolean models and their properties is presented in Section 2. There we define dimension, introduce estimators and describe their properties. The theoretical results discussed in Section 2 are illustrated in a numerical study in Section 3. Outlines of proofs are deferred to Section 4.

A very accessible and concise account of the definition of dimension is given by Taylor and Taylor [(1991), page 354]. See also Barnsley [(1988), page 172ff]. The theory of Boolean models is developed by Ripley (1981), Stoyan, Kendall and Mecke (1987) and Hall (1988), among others. These monographs do not address the topic of the dimension of clusters in a Boolean model, and the notion of a multitype model is not discussed; it appears to be mentioned for the first time in the present paper. Properties of estimators of dimension in more familiar settings have been developed by Ogata and Katsura (1991) and Taylor and Taylor (1991). 


\section{Multitype Boolean models and scaling models.}

2.1. Discrete multitype Boolean disc model. Hermann and Ohser's (1993) discrete multitype Boolean disc model may be described as follows. For each $i \geq 1$, let $\mathscr{Q}_{i}$ denote a homogeneous Poisson process in the plane with intensity $C_{1} \beta^{i}$. At the points of $\mathscr{Q}_{i}$, center discs of radius $C_{2} \alpha^{i}$. We assume that

$$
\alpha<1, \quad \beta>1, \quad \alpha \beta \geq 1, \quad \alpha^{2} \beta<1,
$$

that $C_{1}$ and $C_{2}$ are arbitrary fixed positive constants and that the Poisson point processes $\mathscr{Q}_{i}$ are stochastically independent. Write $\mathscr{C}$ for the resulting coverage process $-\mathscr{C}$ is the collection of discs in the plane and is a special case of the multitype Boolean models that we shall introduce in Section 2.3. The condition $\alpha^{2} \beta<1$ is necessary and sufficient for $\mathscr{C}$ to not cover the entire plane. Indeed, the expected proportion of the plane that is not covered by any discs from $\mathscr{C}$ equals $p=\exp \left\{-\pi C_{1} C_{2}^{2}\left(1-\alpha^{2} \beta\right)^{-1}\right\}$. Since $\alpha \beta \geq 1$, then with probability 1 , each line segment in the plane is cut by an infinite number of disc boundaries from $\mathscr{C}$.

Motivated by Hermann and Ohser's (1993) analysis of rust deposits, one might consider studying a multitype Boolean model at a sequence of different levels of resolution. Suppose that at level $\varepsilon$, only discs of radius $\varepsilon$ or more are visible. Let $n=n(\varepsilon)$ denote the largest integer such that $\alpha^{n} \geq \varepsilon$, and consider this new coverage process of discs; call it $\mathscr{C}_{\varepsilon}$. Draw a tube of thickness $\varepsilon$ around the perimeter of that part of the boundary of the coverage pattern produced by $\mathscr{C}_{\varepsilon}$ which lies within the region $\mathscr{R}$. Let $A(\varepsilon)$ equal the area of this tube. It may be shown that with probability 1 , conditional on $\mathscr{R}$ not being completely covered by discs,

$$
\log A(\varepsilon) / \log \varepsilon \rightarrow L=2+(\log \beta)(\log \alpha)^{-1}
$$

as $\varepsilon \rightarrow 0$; see Theorem 2.1. Therefore we might define the dimension of the multitype Boolean model to be $D=2-L=-(\log \beta) /(\log \alpha)$. Assumption (2.1) guarantees that $1 \leq D<2$, which is the appropriate range for fractal dimension of a curve in the plane.

Result (2.2) is a consequence of the fact that, for small $\varepsilon, A(\varepsilon) \approx$ const. $\varepsilon^{L}$. This should not be interpreted as meaning that the ratio $\rho(\varepsilon)=A(\varepsilon) / \varepsilon^{L}$ converges to a constant as $\varepsilon$ tends to 0 ; it does not, on account of the discrete nature of the function $n(\varepsilon)$. However, with probability $1, \rho(\varepsilon)$ is bounded away from zero and infinity as $\varepsilon$ tends to zero, and that result is sufficient to give (2.2). Furthermore, it is possible to choose a sequence of $\varepsilon$ 's tending to zero such that for a fixed $C>0, A(\varepsilon) / \varepsilon^{L} \rightarrow C$, and, as we shall show in Theorem 2.2, there exist realistic models of particulate processes with a continuum of particle radii, for which $A(\varepsilon) / \varepsilon^{L} \rightarrow C$ with probability 1 as $\varepsilon \rightarrow 0$ along any deterministic sequence. In such cases, (2.2) may be strengthened to

$$
\log A(\varepsilon)-L \log \varepsilon=\log C+o(1)
$$

with probability 1 . 
Another way of interpreting $D$ is as the exponent of $s^{-1}$ in a formula for the intensity of discs of radius $s$ or more. The process of centers of discs of radius greater than or equal to $s$ is homogeneous and Poisson with intensity function

$$
\lambda(s)= \begin{cases}0, & \text { for } s>\alpha, \\ C_{1}\left(\beta^{i+1}-1\right) /(\beta-1), & \text { for } \alpha^{i+1}<s \leq \alpha^{i} .\end{cases}
$$

We call $\lambda$ the cumulative intensity function of the multitype Boolean model. Note particularly that $\lambda$ satisfies $\lambda(s) \asymp s^{-D}$, meaning that the ratio $\lambda(s) / s^{-D}$ is bounded away from 0 and $\infty$ as $s \rightarrow 0$.

While the naive estimator $\tilde{D}=2-\{\log A(\varepsilon)\}(\log \varepsilon)^{-1}$ is consistent for $D$, it is often not of practical use because it is heavily biased. In some instances a more effective estimator may be based on the approximate linear regression obtained by taking logarithms of both sides of the equation $A(\varepsilon) \approx$ const. $\varepsilon^{2-D}$. Indeed, let $\varepsilon_{j}=c_{j} \varepsilon, 1 \leq j \leq k$, where $k \geq 2$ and the constants $c_{j}$ are all positive and distinct, and define $y_{j}=\log c_{j}, \bar{y}=k^{-1} \sum y_{j}, s_{y}^{2}=$ $k^{-1} \sum\left(y_{j}-\bar{y}\right)^{2}$ and $\hat{D}=2-\hat{\theta}$, where

$$
\hat{\theta}=s_{j}^{-2} k^{-1} \sum_{j=1}^{k}\left(y_{j}-\bar{y}\right) \log A\left(\varepsilon_{j}\right) .
$$

Result (2.3) ensures that with probability 1 , conditional on $\mathscr{R}$ not being completely covered, $\hat{D} \rightarrow D$ as $\varepsilon \rightarrow 0$. Alternative approaches to dimension estimation are considered by Cutler (1991) and Serinko (1994). Many generalizations are possible, for example, with weights and with integrals instead of series.

The estimator $\hat{D}$ is really a regression-based version of the well-known box-counting estimators, albeit in an unconventional setting. For accounts of the advantages of adopting a regression approach, see Sullivan and Hunt (1988) and Hunt (1990).

2.2. General multitype Boolean models. Let $\mathscr{P}=\{\mathscr{P}(s), s>0\}$ denote a sequence of marked point process in the plane, with the marks of $\mathscr{P}(s)$ being values of $(r, \mathscr{S})$, where $r \in[s, \infty)$ and $\mathscr{S}$ is a realization of a random set $S$. The point processes are assumed nested, in the sense that $\mathscr{P}\left(s_{2}\right) \subseteq \mathscr{P}\left(s_{1}\right)$ whenever $s_{1}<s_{2}$. With each point $x$ from one of the processes in the sequence $\mathscr{P}$, having mark $(r, \mathscr{S})$, we associate the translated, scaled set $x+r \mathscr{S}=\{x+r y: y \in \mathscr{S}\}$. The general multitype grain model is the collection of all these sets.

The character of a multitype grain model is determined by the nature of the point processes $\mathscr{P}$ and by the way in which marks are distributed among the points. To define a multitype Boolean model in which $\mathscr{P}$ has (cumulative) intensity $\lambda(s)$, for a decreasing function $\lambda$, we ask that for any sequence of positive numbers $0<s_{1}<s_{2}<\cdots<s_{2 n-1}<s_{2 n}$, all the differenced point 
processes $\mathscr{P}\left(s_{2 i-1}\right) \backslash \mathscr{P}\left(s_{2 i}\right), 1 \leq i \leq n$, be stochastically independent Poisson processes with respective intensities $\lambda\left(s_{2 i-1}\right)-\lambda\left(s_{2 i}\right)$. We further assume that points associated with the infinitesimal difference $d \mathscr{P}(r)=\mathscr{P}(r) \backslash$ $\mathscr{P}(r+d r)$ have mark $(r, \mathscr{S})$ for some $\mathscr{S}$ chosen independently and at random from the range space of a random set $S$.

It is perhaps pedantic to insist that the distribution of $S$ in the mark $(r, S)$ not depend on $r$, and indeed it is straightforward to produce versions of our theory for cases where such dependence is allowed. The reason for not doing so is simply to reduce the level of complexity of our analysis, particularly of notation. If we allow $S$ to depend on $r$, then we have to impose assumptions of uniformity on the way in which the dependence alters with varying $r$, and then the notation for describing bias and variance of the estimator $\hat{D}$ becomes much more cumbersome.

We shall argue later in this section that a general multitype Boolean model exhibits the character noted in Section 2.1 and has dimension $D$ if $\lambda(s) \asymp s^{-D}$ as $s \rightarrow 0$. Indeed, the somewhat weaker assumption at (2.6) below is permissible. To obtain precisely the discrete multitype Boolean disc model described in Section 2.1, take $S$ to be the fixed disc of radius $C_{2}$ centered at the origin, let $\mathscr{P}(s)$ be empty if $s>\alpha$ and put $\mathscr{P}(s)=\bigcup_{1 \leq i \leq i_{s}} \mathscr{Q}_{i}$ for $s \leq \alpha$, where $i_{s}$ equals the integer part of $(\log s) /(\log \alpha)$ and the $\mathscr{Q}_{i}$ s are independent Poisson processes with respective intensities $C_{1} \beta^{i}$.

Next we define a version of $A(\varepsilon)$ for the general context. Let $b$ denote any fixed positive number, let $\mathscr{R}$ be any bounded open subset of the plane, let $\mathscr{T}(x, r, \mathscr{S})=x+r \mathscr{S}$ denote one of the translated, scaled sets comprising the model and write $\mathscr{U}(\varepsilon)$ for the union of all sets $\mathscr{T}(x, r, \mathscr{S})$ with $r \geq b \varepsilon$. [The Poisson point at $x$ has mark $(r, \mathscr{S})$.] Define $\mathscr{S}^{\varepsilon}$ to be the set of all points distant $\varepsilon$ or less from $\mathscr{S}$, put $\mathscr{S}_{\varepsilon}=\mathscr{S}^{\varepsilon} \backslash \mathscr{S}$, put $\mathscr{T}(x, r, \mathscr{S}, \varepsilon)=x+(r \mathscr{S})_{\varepsilon}$ and let $A(\varepsilon)$ denote the area of the intersection with $\mathscr{R}$ and the complement of $\mathscr{U}(\varepsilon)$ of the union $\mathscr{V}(\varepsilon)$ of all the sets $\mathscr{T}(x, r, \mathscr{S}, \varepsilon)$ with $r \geq b \varepsilon$. (The latter requirement serves to identify those sets from the original model whose scale is no less than $b \varepsilon$.)

For any measurable subset $\mathscr{S}$ of the plane let $R(\mathscr{S})$ denote the infimum of the radii of all discs that contain $\mathscr{S}$, and write $\|\mathscr{S}\|$ for the area of $\mathscr{S}$. In a slight abuse of notation, if $x$ is a 2 -vector, we write $\|x\|$ for the length (i.e., Euclidean norm) of $x$. In stating our strong law for $\log A(\varepsilon) / \log \varepsilon$, we suppose that for some $C>0, P\{R(S)>C\}=0$, that is $R(S)$ is essentially bounded, and that $P\left(\left\|S^{\varepsilon} \backslash S\right\|>C \varepsilon\right)=0$ for all $\varepsilon>0$. The boundedness condition may be relaxed by employing a longer proof involving a truncation argument, but we shall not elaborate that point further here. In place of $\lambda(s) \asymp s^{-D}$ we impose the weaker condition that for some $1<D<2$,

$$
\begin{gathered}
\text { for all } \delta>0, \quad \lambda(s)=O\left(s^{-D-\delta}\right) \quad \text { and } \\
s^{-D+\delta}=O\{\lambda(s)\} \quad \text { as } s \rightarrow 0 .
\end{gathered}
$$

We shall say that $\mathscr{R}$ is not completely covered by the multitype Boolean model if the area of that subset of $\mathscr{R}$ which is not covered is strictly positive. 
THEOREM 2.1. Assume that sets are laid down according to a multitype Boolean model satisfying the conditions announced in the previous paragraph. Then with probability 1 , conditional on $\mathscr{R}$ not being completely covered,

$$
\lim _{\varepsilon \rightarrow 0} \log A(\varepsilon) / \log \varepsilon=2-D \text {. }
$$

Should (2.4) hold for a value of $D$ which lies outside the range $(1,2)$, then it is generally inappropriate to interpret that $D$ as dimension. This is most clearly seen in the case of the discrete disc model of Section 2.1. There, Minkowski dimension and capacity dimension are well defined and equal 1 when $\alpha \beta<1$, but the formula $D(\alpha, \beta)=-(\log \beta) /(\log \alpha)$ suggests that $D<1$. Assuming the conditions in (2.1) except for $\alpha \beta \geq 1$, it may be shown that in the discrete disc model the formula for $D(\alpha, \beta)$ is appropriate if and only if $\alpha \beta \geq 1$ and $\alpha^{2} \beta<1$.

Statistical consistency of the estimator $\tilde{D}$ follows from Theorem 2.1. That result may be derived as a corollary of a more refined theorem for a multitype Boolean model with a continuous, regularly varying cumulative intensity function. To describe such a result, first define a particular cumulative intensity function,

$$
\lambda(s)=\int_{s}^{\infty} r^{-a} f(r) d r
$$

where $2<a<3$ and $f \geq 0$ is bounded on $(r, \infty)$ for each $r>0$, is slowly varying at the origin and satisfies $f(r)=O\left(r^{a-6}\right)$ as $r \rightarrow \infty$. [See Bingham, Goldie and Teugels (1987) for extensive discussion of slow and regular variation.] Let $\mathscr{R}$ be as before, define $\pi$ to equal the proportion of $\mathscr{R}$ not covered by the multitype Boolean model and put

and

$$
p=E(\pi)=\exp \left\{-E\|S\| \int_{0}^{\infty} r^{2-a} f(r) d r\right\}
$$

$$
C_{0}=\|\mathscr{R}\| p \int_{b}^{\infty} E\left\|S^{1 / r} \backslash S\right\| r^{2-a} d r
$$

Consistency of $\hat{D}$ for $D$ follows from the following result, noting that it implies (2.3).

Theorem 2.2. Assume the conditions of Theorem 2.1, except that condition (2.4) on $\lambda$ is replaced by asking that $\lambda$ admit the definition at (2.5), where $a$ and $f$ satisfy the conditions stated in the previous paragraph. Then $E A(\varepsilon) \sim$ $C_{0} \varepsilon^{3-a} f(\varepsilon)$ and, with probability $1, A(\varepsilon) / E A(\varepsilon) \rightarrow \pi / p$, both results holding as $\varepsilon \rightarrow 0$.

\subsection{Properties of $\hat{D}$.}

2.3.1. Preliminaries. As a prelude to describing the main properties of $\hat{\theta}$, and hence of $\hat{D}$, let $\pi$ [respectively, $\pi(\varepsilon)$ ] denote the proportion of $\mathscr{R}$ covered by the multitype Boolean model [by those sets in the model whose centers 
have mark $(r, \mathscr{S})$ satisfying $r \geq b \varepsilon]$. Put $p=E(\pi), p(\varepsilon)=E\{\pi(\varepsilon)\}, \pi_{j}=$ $\pi\left(\varepsilon_{j}\right), p_{j}=p\left(\varepsilon_{j}\right)$ and $Q_{j}=\left\{A\left(\varepsilon_{j}\right)-\left(\pi_{j} / p_{j}\right) E A\left(\varepsilon_{j}\right)\right\} / E A\left(\varepsilon_{j}\right)$, and observe that if $\pi>0$, then, as $\varepsilon \rightarrow 0$,

$$
\hat{\theta}=\theta_{\varepsilon}+\hat{\Theta}_{\varepsilon}+O_{p}\left[\sum_{j=1}^{k}\left\{Q_{j}^{2}+\left(p \pi_{j}-p_{j} \pi\right)^{2}\right\}\right],
$$

where

$$
\begin{aligned}
& \theta_{\varepsilon}=s_{y}^{-2} k^{-1} \sum_{j=1}^{k}\left(y_{j}-\bar{y}\right) \log \left\{E A\left(\varepsilon_{j}\right)\right\} \\
& \hat{\Theta}_{\varepsilon}=s_{y}^{-2} k^{-1} \sum_{j=1}^{k}\left(y_{j}-\bar{y}\right)\left\{\left(p_{j} / \pi_{j}\right) Q_{j}+p_{j}^{-1}\left(\pi_{j}-p_{j}\right)\right\} .
\end{aligned}
$$

The term $\theta_{\varepsilon}$ is the major contributor to bias; the term $\hat{\Theta}_{\varepsilon}$ is the major contributor to error about the mean.

2.3.2. Size of $\theta_{\varepsilon}$. We ask that the cumulative intensity function, $\lambda$, be expressible by (2.5), where as $r \rightarrow 0$,

$$
f(r)=\alpha_{10}+\alpha_{11} r^{a-a_{1}}+o\left(r^{a-a_{1}}\right) .
$$

In this formula, $2<a_{1}<a<3,0<\alpha_{10}<\infty,-\infty<\alpha_{11}<\infty$. Put

$$
\begin{gathered}
\alpha_{1}=\frac{\alpha_{11} \int_{b}^{\infty} E\left\|S^{1 / r} \backslash S\right\| r^{2-a_{1}} d r}{\alpha_{10} \int_{b}^{\infty} E\left\|S^{1 / r} \backslash S\right\| r^{2-a} d r}, \\
\alpha_{2}=\alpha_{10} \frac{\int_{b}^{\infty} E\left\|S^{1 / t} \backslash S\right\| t^{2-a}\left\{E\|S\|(3-a)^{-1} b^{3-a}-\int_{t}^{\infty} E\left\|S^{1 / t} \backslash S\right\| r^{2-a} d r\right\} d t}{\int_{b}^{\infty} E\left\|S^{1 / r} \backslash S\right\| r^{2-a} d r} \\
t_{0}=\alpha_{2} s_{y}^{-2} k^{-1} \sum_{j=1}^{k}\left(y_{j}-\bar{y}\right) c_{j}^{3-a}, \quad t_{1}=\alpha_{1} s_{y}^{-2} k^{-1} \sum_{j=1}^{k}\left(y_{j}-\bar{y}\right) c_{j}^{a-a_{1}} .
\end{gathered}
$$

It is proved by Hall and Polzehl (1994) that, assuming (2.7),

$$
\theta_{\varepsilon}+D-2=t_{0} \varepsilon^{3-a}+t_{1} \varepsilon^{a-a_{1}}+o\left(\varepsilon^{3-a}+\varepsilon^{a-a_{1}}\right) .
$$

2.3.3. Size of $\hat{\Theta}_{\varepsilon}$. We assume the conditions of Theorem 2.2, with $f(r) \rightarrow$ $\alpha_{10}>0$ as $r \rightarrow 0$, and that there exist functions $g$ and $g_{0}$ such that

$$
\begin{aligned}
\varepsilon^{-1} E\left\|\left(S^{\varepsilon} \backslash S\right) \cap(x+S)\right\| & \rightarrow g(x), \\
\varepsilon^{-2} E\left\|\left(S^{\varepsilon} \backslash S\right) \cap\left\{x+\left(S^{\varepsilon} \backslash S\right)\right\}\right\| & \leq g_{0}(x),
\end{aligned}
$$


where $\int g_{0}<\infty$. [The assumptions of Theorem 2.2 imply that $g(x) \leq C_{1} I(\|x\|$ $\leq C_{2}$ ), for constants $C_{1}, C_{2}>0$. The reasonableness of the displayed conditions is discussed by Hall and Polzehl (1994).] Define

$$
\begin{aligned}
Q(\varepsilon) & =[A(\varepsilon)-\{\pi(\varepsilon) / p(\varepsilon)\} E A(\varepsilon)] / E A(\varepsilon), \\
N(\varepsilon) & =\{p p(\varepsilon)\}^{-1}\{p \pi(\varepsilon)-p(\varepsilon) \pi\},
\end{aligned}
$$

in which notation

$$
\hat{\Theta}_{\varepsilon}=s_{y}^{-2} k^{-1} \sum_{j=1}^{k}\left(y_{j}-\bar{y}\right)\left\{\left(p_{j} / \pi_{j}\right) Q\left(\varepsilon_{j}\right)+\pi^{-1} N\left(\varepsilon_{j}\right)\right\} .
$$

It is shown by Hall and Polzehl (1994) that for constants $B_{1}, B_{2}>0$,

$$
E\left\{Q(\varepsilon)^{2}\right\} \sim B_{1} \varepsilon^{2}=o\left(\varepsilon^{2(3-a)}\right), \quad E\left\{N(\varepsilon)^{2}\right\} \sim B_{2} \varepsilon^{5-a}=o\left(\varepsilon^{2(3-a)}\right) .
$$

Therefore, $E\left(\hat{\Theta}_{\varepsilon}^{2}\right)=o\left\{\left(D-2+\theta_{\varepsilon}\right)^{2}\right\}$; compare (2.8) and (2.9).

2.3.4. Implications of formulae for $\theta_{\varepsilon}$ and $E\left(\hat{\Theta}_{\varepsilon}^{2}\right)$. Combining results from Sections 2.3.1-2.3.3 we see that one may write

$$
\hat{D}=D-\left(t_{0} \varepsilon^{3-a}+t_{1} \varepsilon^{a-a_{1}}\right)+o_{p}\left(\varepsilon^{3-a}+\varepsilon^{a-a_{1}}\right) .
$$

This provides a particularly detailed account of the rate of convergence of $\hat{D}$ to $D$.

3. Numerical results. To investigate properties of the proposed estimators, we ran simulations for various setups of parameters, differently shaped patterns and varying resolution levels. In the first study we used the discrete multitype Boolean disc model described in Section 2.1. A value of $\beta=1.001$ was employed in order to obtain a process approximating a continuous Boolean model. The dimension $D$, the proportion $p$ not covered by pattern and the maximal disc radius $C_{2}$ were fixed in order to describe different situations. We obtained $\alpha$ and $C_{1}$ using the equations for $p$ and $D$ given in Section 2.1. Write $\hat{D}_{k}$ for $\hat{D}$ to indicate dependence on $k$-point linear regression $\left(c_{j}=j\right)$. Results are given in terms of bias, standard deviation (s.d.) and mean squared error (mse) of estimators $\hat{D}_{k}$. We approximated $A(\varepsilon)$ by Monte Carlo methods, using $5 \times 10^{5}$ random points. This restricted the contribution of the approximation error to no more than $25 \%$ of the variance of $\hat{D}$ in worst possible situations.

Table 1 summarizes, in the case $p=0.5$, a much larger simulation study of the relative performance of $\tilde{D}$ and $\hat{D}_{2}, \ldots, \hat{D}_{5}$. The results there show that both the bias and variance of $\hat{D}_{k}$ increase with increasing $k$. The increase in variance is a consequence of strong positive correlation between values of $\log A\left(\varepsilon_{k}\right)$.

We also examined the effect of set shape. Initially we replaced the discs by ellipses of equal area in the coverage process. The ellipses were chosen to have either random (uniform) orientation or fixed orientation (larger half-axis in the horizontal direction). Eccentricities were taken to have a logarithmic Normal distribution with logarithmic mean $\mu$ and logarithmic standard 
TABLE 1

Bias, standard deviation (s.d.) and mean squared error (mse) of estimators $\hat{D}_{2}$ and $\hat{D}_{5}$

\begin{tabular}{|c|c|c|c|c|c|c|c|c|}
\hline \multirow[b]{2}{*}{$D$} & \multirow[b]{2}{*}{$C_{2}$} & \multirow[b]{2}{*}{$\varepsilon$} & \multicolumn{2}{|c|}{ Bias } & \multicolumn{2}{|c|}{ s.d. } & \multicolumn{2}{|c|}{ mse } \\
\hline & & & $\hat{D}_{2}$ & $\hat{D}_{5}$ & $\hat{D}_{2}$ & $\hat{D}_{5}$ & $\hat{D}_{2}$ & $\hat{D}_{5}$ \\
\hline \multirow[t]{2}{*}{1.1} & 0.4 & 0.001 & 0.128 & 0.142 & 0.045 & 0.044 & 0.0185 & 0.022 \\
\hline & & 0.01 & 0.242 & 0.292 & 0.114 & 0.134 & 0.0715 & 0.1035 \\
\hline \multirow[t]{2}{*}{1.1} & 0.2 & 0.001 & 0.151 & 0.168 & 0.029 & 0.026 & 0.0235 & 0.0288 \\
\hline & & 0.01 & 0.294 & 0.352 & 0.092 & 0.1 & 0.0951 & 0.1342 \\
\hline \multirow[t]{2}{*}{1.1} & 0.1 & 0.423 & 0.183 & 0.206 & 0.024 & 0.023 & 0.0342 & 0.043 \\
\hline & & 0.01 & 0.387 & 0.547 & 0.084 & 0.11 & 0.1571 & 0.3109 \\
\hline \multirow[t]{2}{*}{1.25} & 0.4 & 0.001 & 0.075 & 0.084 & 0.049 & 0.048 & 0.0081 & 0.0094 \\
\hline & & 0.01 & 0.149 & 0.19 & 0.112 & 0.118 & 0.0348 & 0.05 \\
\hline \multirow[t]{2}{*}{1.25} & 0.2 & 0.001 & 0.093 & 0.108 & 0.03 & 0.032 & 0.0095 & 0.0127 \\
\hline & & 0.01 & 0.207 & 0.263 & 0.082 & 0.11 & 0.0495 & 0.0815 \\
\hline \multirow[t]{2}{*}{1.25} & 0.1 & 0.001 & 0.114 & 0.134 & 0.02 & 0.02 & 0.0135 & 0.0183 \\
\hline & & 0.01 & 0.292 & 0.463 & 0.077 & 0.12 & 0.0914 & 0.2288 \\
\hline \multirow[t]{2}{*}{1.5} & 0.4 & 0.001 & 0.007 & 0.014 & 0.038 & 0.042 & 0.0015 & 0.002 \\
\hline & & 0.01 & 0.053 & 0.092 & 0.142 & 0.167 & 0.0229 & 0.0364 \\
\hline \multirow[t]{2}{*}{1.5} & 0.2 & 0.001 & 0.02 & 0.029 & 0.031 & 0.034 & 0.0014 & 0.002 \\
\hline & & 0.01 & 0.113 & 0.179 & 0.121 & 0.16 & 0.0274 & 0.0576 \\
\hline \multirow[t]{2}{*}{1.5} & 0.1 & 0.001 & 0.031 & 0.044 & 0.021 & 0.024 & 0.0014 & 0.0025 \\
\hline & & 0.01 & 0.197 & 0.364 & 0.101 & 0.115 & 0.0491 & 0.1454 \\
\hline \multirow[t]{2}{*}{1.75} & 0.4 & 0.001 & -0.03 & -0.033 & 0.028 & 0.039 & 0.0017 & 0.0026 \\
\hline & & 0.1 & 0.003 & 0.036 & 0.172 & 0.198 & 0.0294 & 0.0406 \\
\hline \multirow[t]{2}{*}{1.75} & 0.2 & 0.001 & -0.029 & -0.022 & 0.023 & 0.029 & 0.0013 & 0.0013 \\
\hline & & 0.01 & 0.047 & 0.144 & 0.131 & 0.21 & 0.0194 & 0.0649 \\
\hline \multirow[t]{2}{*}{1.75} & 0.1 & 0.001 & -0.027 & -0.02 & 0.022 & 0.023 & 0.0012 & 0.0009 \\
\hline & & 0.01 & 0.107 & 0.318 & 0.125 & 0.183 & 0.0271 & 0.1343 \\
\hline
\end{tabular}

deviation $\mu^{1 / 2}$. The ellipses were assigned to $\mathscr{C}_{\varepsilon}$ if the smaller half-axis exceeded $\varepsilon$. Figure 1 illustrates the coverage process at level $\varepsilon=0.001$ for parameters $D=1.5, p=0.5, C_{2}=0.1, \beta=1.001$ and $\mu=1$. Results are given in Table 2 for random orientation. Details of the case of fixed orientation, of the effect of resolution level misspecification and of other matters are available from Hall and Polzehl (1994).

4. Outline proofs of Theorems 2.1 and 2.2. [Details are available from Hall and Polzehl (1994).] Since Theorem 2.1 may be derived as a corollary of Theorem 2.2 , we prove the latter first.

OUtLINE PROOF OF THEOREM 2.2. Let $\varepsilon_{1}, \varepsilon_{2}, \ldots$ denote a strictly decreasing sequence of positive numbers such that $\varepsilon_{i} \rightarrow 0$ and

$$
\left\{\inf _{(i)} E A(\varepsilon)\right\} /\left\{\sup _{(i)} E A(\varepsilon)\right\} \rightarrow 1
$$




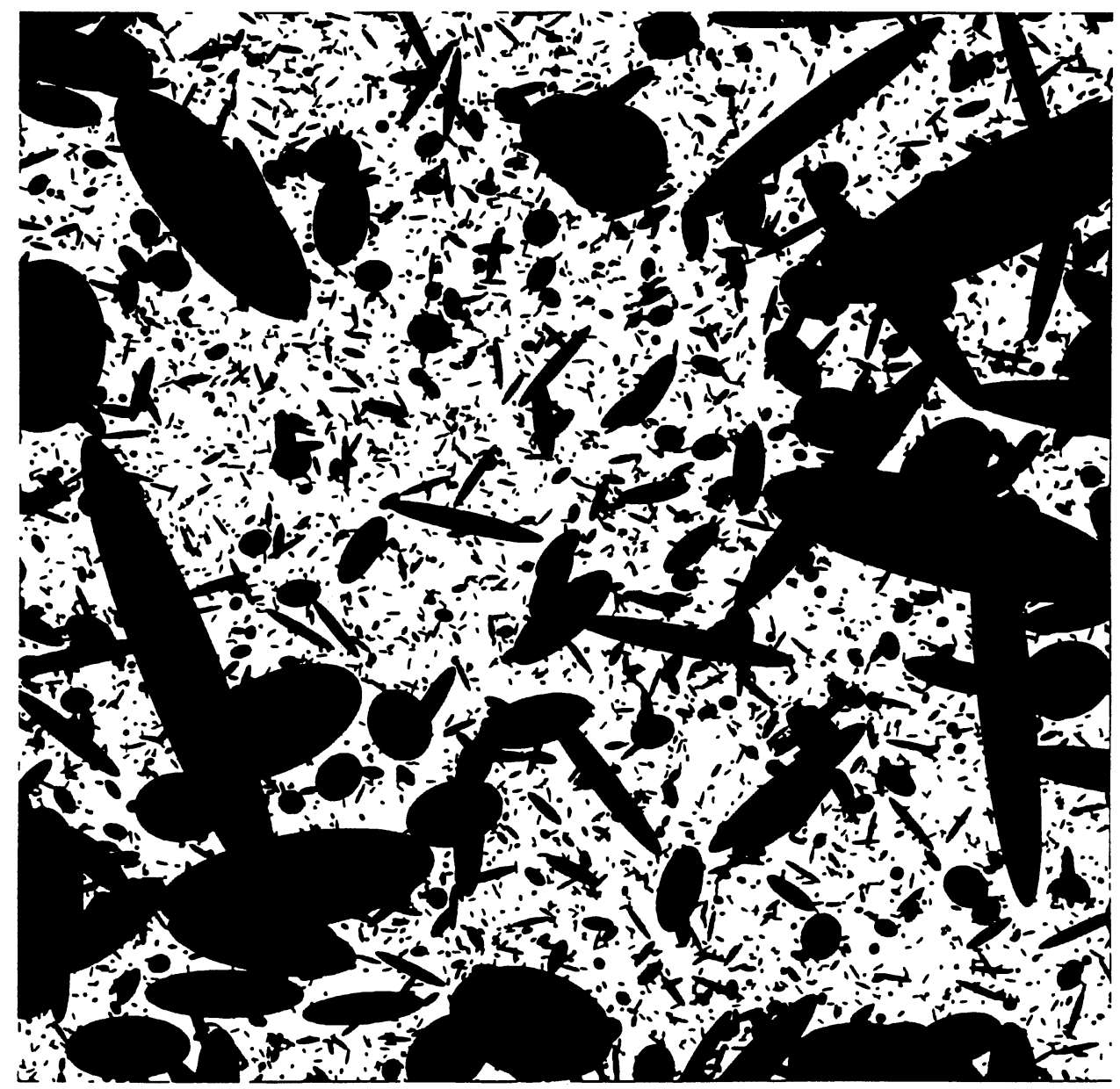

FIG. 1. Multitype Boolean ellipse model $(D=1.5, p=0.5)$.

as $i \rightarrow \infty$, where $\inf _{(i)}$ and $\sup _{(i)}$ denote, respectively, the infimum and supremum over $\varepsilon \in\left[\varepsilon_{i+1}, \varepsilon_{i}\right]$. It suffices to show that

$$
\begin{aligned}
& \left\{A\left(\varepsilon_{i}\right)-(\pi / p) E A\left(\varepsilon_{i}\right)\right\} / E A\left(\varepsilon_{i}\right) \rightarrow 0, \\
& \left\{\sup _{(i)}\left|A(\varepsilon)-A\left(\varepsilon_{i+1}\right)\right|\right\} / E A\left(\varepsilon_{i}\right) \rightarrow 0
\end{aligned}
$$

with probability 1 .

Given $0<\varepsilon^{\prime}<\varepsilon$, write $B_{1}\left(\varepsilon, \varepsilon^{\prime}\right)$ for the area of the region within $\mathscr{R}$ covered by sets $x+r\left(\mathscr{S}^{\varepsilon} \backslash \mathscr{S}^{\varepsilon^{\prime}}\right)$, where $x \in \mathscr{P}(b \varepsilon)$ has mark $(r, \mathscr{S})$ for some $r \geq b \varepsilon$, and write $B_{2}\left(\varepsilon, \varepsilon^{\prime}\right)$ for the area of the region within $\mathscr{R}$ covered by sets $x+r\left(\mathscr{S}^{\varepsilon^{\prime}} \backslash \mathscr{S}\right)$, where $x \in \mathscr{P}\left(b \varepsilon^{\prime}\right) \backslash \mathscr{P}(b \varepsilon)$ has mark $(r, \mathscr{S})$ for some $r \in$ 
TABLE 2

Effects of shape on $\hat{D}_{2}$ (ellipses with random orientation)

\begin{tabular}{|c|c|c|c|c|c|c|c|}
\hline \multirow[b]{2}{*}{$\boldsymbol{D}$} & \multirow[b]{2}{*}{$\boldsymbol{\mu}$} & \multicolumn{2}{|c|}{$\operatorname{Bias}\left(\hat{D}_{2}\right)$} & \multicolumn{2}{|c|}{ s.d. $\left(\hat{D}_{2}\right)$} & \multicolumn{2}{|c|}{$\operatorname{mse}\left(\hat{D}_{2}\right)$} \\
\hline & & $\varepsilon=0.001$ & $\bar{\varepsilon}=\mathbf{0 . 0 1}$ & $\varepsilon=0.001$ & $\bar{\varepsilon}=\mathbf{0 . 0 1}$ & $\bar{\varepsilon}=\mathbf{0 . 0 0 1}$ & $\varepsilon=0.01$ \\
\hline 1.1 & 0.5 & 0.136 & 0.223 & 0.051 & 0.132 & 0.021 & 0.0673 \\
\hline 1.1 & 1 & 0.147 & 0.269 & 0.053 & 0.136 & 0.0245 & 0.0908 \\
\hline 1.25 & 0.5 & 0.08 & 0.186 & 0.047 & 0.16 & 0.0086 & 0.0602 \\
\hline 1.25 & 1 & 0.083 & 0.185 & 0.051 & 0.178 & 0.0095 & 0.0659 \\
\hline 1.5 & 0.5 & 0.017 & 0.085 & 0.046 & 0.16 & 0.0024 & 0.0327 \\
\hline 1.5 & 1 & 0.018 & 0.117 & 0.043 & 0.197 & 0.0022 & 0.0526 \\
\hline 1.75 & 0.5 & -0.038 & 0.022 & 0.041 & 0.224 & 0.0031 & 0.0505 \\
\hline 1.75 & 1 & -0.028 & 0.057 & 0.041 & 0.214 & 0.0025 & 0.0491 \\
\hline
\end{tabular}

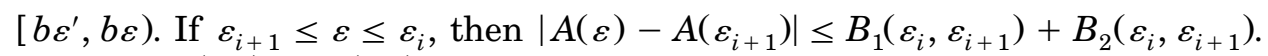
Therefore, (4.2) and (4.3) will follow if we prove that

$$
\begin{gathered}
\left\{E B_{1}\left(\varepsilon_{i}, \varepsilon_{i+1}\right)+E B_{2}\left(\varepsilon_{i}, \varepsilon_{i+1}\right)\right\} / E A\left(\varepsilon_{i}\right) \rightarrow 0, \\
\left\{A\left(\varepsilon_{i}\right)-(\pi / p) E A\left(\varepsilon_{i}\right)\right\} / E A\left(\varepsilon_{i}\right) \rightarrow 0, \quad\left\{D_{i}-E D_{i}\right\} / E A\left(\varepsilon_{i}\right) \rightarrow 0
\end{gathered}
$$

with probability 1 , where $D_{i}=B_{1}\left(\varepsilon_{i}, \varepsilon_{i+1}\right)$ or $B_{2}\left(\varepsilon_{i}, \varepsilon_{i+1}\right)$.

Recall the definitions of $\mathscr{T}(x, r, \mathscr{S})$ and $\mathscr{T}(x, r, \mathscr{S}, \varepsilon)$ given three paragraphs above Theorem 2.1. Given $t \geq b \varepsilon$, let $\mathscr{U}(\varepsilon, t)$ denote the union of all those sets $\mathscr{T}(x, r, \mathscr{S})$ with $b \varepsilon \leq r<t$, write $\mathscr{V}(\varepsilon, t)$ for the union of all those sets $\mathscr{T}(x, r, \mathscr{S}, \varepsilon)$ with $r>t$ and let $\mathscr{W}(\varepsilon, t)$ be the union of all those sets $\mathscr{T}(x, r, \mathscr{S}) \cup \mathscr{T}(x, r, \mathscr{S}, \varepsilon)$ with $r>t$. Put $\mathscr{A}=\mathscr{U}(\varepsilon, \infty)^{\sim} \cap \mathscr{V}(\varepsilon, b \varepsilon)$, where the tilde denotes complementation. Note that $A(\varepsilon)$ equals the area of $\mathscr{A} \cap \mathscr{R}$. Therefore, $E A(\varepsilon)=q_{0}\|\mathscr{R}\|$, where $q_{0}=P(O \in \mathscr{W})$ and $O$ denotes the origin in the plane. It may be proved that

$$
q_{0} \sim\|\mathscr{R}\|^{-1} C_{0} \varepsilon^{3-a} f(\varepsilon),
$$

whence it follows that $E\{A(\varepsilon)\} \sim C_{0} \varepsilon^{3-a} f(\varepsilon)$. Here $C_{0}$ is as defined in Section 2. Therefore, (4.1) will follow if the $\varepsilon_{i}$ 's are chosen such that

$$
\varepsilon_{i} / \varepsilon_{i+1} \rightarrow 1
$$

as $i \rightarrow \infty$. Similarly it may be proved that (4.7) is sufficient for (4.4). Result (4.5) will follow via the Borel-Cantelli lemma if we show that for all $\eta>0$,

$$
\begin{gathered}
\sum_{i=1}^{\infty} P\left\{\left|A\left(\varepsilon_{i}\right)-(\pi / p) E A\left(\varepsilon_{i}\right)\right|>\eta E A\left(\varepsilon_{i}\right)\right\} \\
+\sum_{i=1}^{\infty} P\left\{\left|D_{i}-E D_{i}\right|>\eta E A\left(\varepsilon_{i}\right)\right\}<\infty .
\end{gathered}
$$

We shall consider only the finiteness of the first series in (4.8).

Let $0<\xi<1$ be fixed, define the intervals $\mathscr{I}_{1}=\left[\varepsilon^{\xi}, \infty\right)$ and $\mathscr{I}_{2}=\left[b \varepsilon, \varepsilon^{\xi}\right)$ and for a general interval $\mathscr{I}$ let $\mathscr{Z}_{1}(\mathscr{I})$ [respectively, $\left.\mathscr{Z}_{2}(\mathscr{I})\right]$ denote the union 
of sets $\mathscr{T}(x, r, \mathscr{S})[\mathscr{T}(x, r, \mathscr{S}, \varepsilon)]$ with $r \in \mathscr{I}$. Put $\mathscr{X}_{i}=\mathscr{Z}_{1}\left(\mathscr{F}_{i}\right)$ and $\mathscr{Y}_{i}=\mathscr{Z}_{2}\left(\mathscr{I}_{i}\right)$, $A_{1}(\varepsilon)=\left\|\mathscr{R} \cap \mathscr{X}_{1}^{\sim} \cap \mathscr{Y}_{2}\right\|, A_{2}(\varepsilon)=\left\|\mathscr{R} \cap \mathscr{Y}_{1}\right\|, A_{3}(\varepsilon)=\left\|\mathscr{R} \cap \mathscr{X}_{2} \cap \mathscr{Y}_{2}\right\|$. Then

$$
\begin{aligned}
\mid A(\varepsilon) & -(\pi / p) E A(\varepsilon)-\left\{A_{1}(\varepsilon)-(\pi / p) E A_{1}(\varepsilon)\right\} \mid \\
\leq & \sum_{j=2}^{3}\left\{A_{j}(\varepsilon)+E A_{j}(\varepsilon)\right\} .
\end{aligned}
$$

Let $C_{1}, C_{2}, \ldots$ denote generic positive constants. It may be proved that $E\left\{A_{2}(\varepsilon)\right\} \leq C_{1} \varepsilon^{1+\xi(2-a)} f\left(\varepsilon^{\xi}\right)$ and $E\left\{A_{3}(\varepsilon)\right\} \leq C_{2} \varepsilon^{(3-a)(1+\xi)}\left\{f(\varepsilon)+f\left(\varepsilon^{\xi}\right)\right\}^{2}$. From these results and (4.6) it follows that $E\left\{A_{j}(\varepsilon)\right\} / E\{A(\varepsilon)\} \rightarrow 0$ for $j=2$ and 3. Hence, in view of (4.9), to show that the first series in (4.8) is finite when $D_{i}=A\left(\varepsilon_{i}\right)$ it suffices to show that for all $\eta>0$ and $j=2$ and 3 ,

$$
\begin{gathered}
\sum_{i=1}^{\infty} P\left\{A_{j}\left(\varepsilon_{i}\right)>\eta \varepsilon_{i}^{3-a} f\left(\varepsilon_{i}\right)\right\}<\infty, \\
\sum_{i=1}^{\infty} P\left\{\left|A_{1}\left(\varepsilon_{i}\right)-(\pi / p) E A_{1}\left(\varepsilon_{i}\right)\right|>\eta \varepsilon_{i}^{3-a} f\left(\varepsilon_{i}\right)\right\}<\infty .
\end{gathered}
$$

To derive (4.10) for $j=2$, observe that $A_{2}(\varepsilon) \leq C_{3} \varepsilon N\left(\varepsilon^{\xi}\right)$, where $N(r)$ equals the number of points in $\mathscr{P}(r)$ that are within ess $\sup R(S)+1$ of the boundary of $\mathscr{R}$. Therefore, $P\left\{A_{2}(\varepsilon)>\eta \varepsilon^{3-a} f\left(\varepsilon^{\xi}\right)\right\} \leq P\left\{N\left(\varepsilon^{\xi}\right)>\right.$ $\left.C_{3}^{-1} \eta \varepsilon^{2-a} f\left(\varepsilon^{\xi}\right)\right\}$. Furthermore, $E\left\{N\left(\varepsilon^{\xi}\right)\right\}>C_{4} \varepsilon^{\xi(1-a)} f\left(\varepsilon^{\xi}\right)$. Hence, if $0<\xi<$ $(a-2) /(a-1)$, then large deviation theory for the Poisson distribution may be used to prove that for all $\lambda>0$ and $j=2$,

$$
P\left\{A_{j}(\varepsilon)>\eta \varepsilon^{3-a} f\left(\varepsilon^{\xi}\right)\right\} \leq C_{5}(\lambda) \varepsilon^{\lambda} .
$$

This proves (4.10), provided that for some $\lambda>0$,

$$
\sum_{i=1}^{\infty} \varepsilon_{i}^{\lambda}<\infty
$$

Similarly, (4.10) for $j=3$ follows from (4.13), after establishing (4.12) in that case.

Next we derive (4.11). Let $\mathscr{F}$ denote the $\sigma$-field defined by the process of sets $\mathscr{T}(x, r, \mathscr{S})$ with $r \in\left[\varepsilon^{\xi}, \infty\right)$, and write $E^{\prime}$ for expectation conditional on $\mathscr{F}$. Assume, without loss of generality, that ess sup $R(S) \leq 1$. Divide $\mathscr{R}$ into a lattice of squares of edge width $4 \varepsilon^{\xi}$. (Of course, toward the perimeter of $\mathscr{R}$ the squares will be only partly within $\mathscr{R}$.) We may distribute the squares among four classes in such a manner that for the $j$ th class, no two squares are closer than $4 \varepsilon^{\xi}$ at their nearest point and the total number of squares in that class equals $m_{j} \sim C_{6} \varepsilon^{-2 \xi}$ as $\varepsilon \rightarrow 0$. Write $A_{(j)}(\varepsilon)$ for the contribution to $A_{1}(\varepsilon)$ from squares in the $j$ th class and let $A_{(j, k)}, 1 \leq k \leq m_{j}$, be the contribution to $A_{(j)}(\varepsilon)$ from the $k$ th square in the $j$ th class. Then for $k_{1} \neq k_{2}$, 
and conditional on $\mathscr{F}, A_{\left(j, k_{1}\right)}(\varepsilon)$ is independent of $A_{\left(j, k_{2}\right)}(\varepsilon)$. Let $t>0$ and let $n \geq 2$ be an even integer. In view of the properties just noted,

$$
\begin{aligned}
\alpha & \equiv P\left\{\left|A_{1}(\varepsilon)-(\pi / p) E A_{1}(\varepsilon)\right|>5 t\right\} \leq t^{-n}\left(\sum_{j=1}^{4} \alpha_{1 j}+\alpha_{2}\right), \\
\alpha_{1 j} & =E\left\{\left|A_{(j)}(\varepsilon)-E^{\prime} A_{(j)}(\varepsilon)\right|^{n}\right\}, \\
\alpha_{2} & =E\left\{\left|E^{\prime} A_{1}(\varepsilon)-(\pi / p) E A_{1}(\varepsilon)\right|^{n}\right\} .
\end{aligned}
$$

To bound $\alpha_{1 j}$, observe that by the conditional independence noted above,

$$
\begin{array}{r}
\alpha_{1 j} \leq C_{7}(n)\left\{E\left(\left[\sum_{k=1}^{m_{j}} E^{\prime}\left\{\left|A_{(j, k)}(\varepsilon)-E^{\prime} A_{(j, k)}(\varepsilon)\right|^{2}\right\}\right]^{n / 2}\right)\right. \\
\left.+\sum_{k=1}^{m_{j}} E\left|A_{(j, k)}(\varepsilon)-E^{\prime} A_{(j, k)}(\varepsilon)\right|^{n}\right\},
\end{array}
$$

from which it may be proved that

$$
\sum_{j=1}^{4} \alpha_{1 j} \leq C_{8}(n) \varepsilon^{n\{1+(1 / 2) \xi(3-a)\}} .
$$

To bound $\alpha_{2}$, define $\pi^{\prime}$ to equal the proportion of $\mathscr{R}$ covered by $\mathscr{X}_{1}$ and put

$$
u=1-\exp \left\{-\int_{b \varepsilon}^{\varepsilon^{\xi}} E\left\|S^{\varepsilon / r} \backslash s\right\| r^{2-a} f(r) d r\right\}=O\left\{\varepsilon^{3-a} f(\varepsilon)\right\},
$$

$v=E\left(\pi^{\prime}\right)$ and $\pi^{\prime \prime}=(\pi / p) v$. Then $E^{\prime}\left\{A_{1}(\varepsilon)\right\}-(\pi / p) E\left\{A_{1}(\varepsilon)\right\}=\pi^{\prime} u-$ $(\pi / p) u v=\left(\pi^{\prime}-\pi^{\prime \prime}\right) u$ and so

$$
\alpha_{2} \leq C_{9}(n)\left\{\varepsilon^{3-a} f(\varepsilon)\right\}^{n} E\left(\pi^{\prime}-\pi^{\prime \prime}\right)^{2 n} \leq C_{10}(n)\left\{\varepsilon^{3-a} f(\varepsilon)\right\}^{n} \varepsilon^{\lambda},
$$

where $\lambda=\lambda(n)$ may be chosen arbitrarily large by taking $n$ sufficiently large. that

Taking $t=\frac{1}{5} \eta \varepsilon^{3-a} f(\varepsilon)$ in (4.14) and noting (4.16) and (4.17), we deduce

$$
\begin{aligned}
& P\left\{\left|A_{1}(\varepsilon)-E A_{1}(\varepsilon)\right|>\eta \varepsilon^{3-a} f(\varepsilon)\right\} \\
& \quad \leq C_{11}(n, \eta)\left[\left\{\varepsilon^{1-\{1-(1 / 2) \xi\}(3-a)} f(\varepsilon)^{-1}\right\}^{n}+\varepsilon^{\lambda}\right],
\end{aligned}
$$

which, since $2<a<3$, equals $O\left(\varepsilon^{\lambda}\right)$ if $n$ is sufficiently large. Therefore, (4.11) follows from (4.13). It remains only to determine decreasing constants $\varepsilon_{i}$ such that (4.7) and (4.13) hold. A suitable choice is $\varepsilon_{i}=i^{-C}$, for arbitrary $C>0$.

Outline PROOF OF THeOREM 2.1. Let $\lambda$ denote a cumulative intensity function satisfying (2.4) and assume initially that $\lambda$ vanishes on $(1, \infty)$. Let $\mathscr{C}$ be a multitype Boolean model corresponding to this particular intensity function. Define $a=D+1$, let $\eta \in(0, \min (D-1,2-D))$ denote an arbitrarily small positive number and choose $0<B_{1}<B_{2}<\infty$, depending on $\eta$, 
in such a way that $\lambda_{1}(s) \equiv B_{1} s^{-D+\eta} \leq \lambda(s) \leq \lambda_{2}(s) \equiv B_{2} s^{-D-\eta}$ for all $s \in$ $(0,1]$. Define $\lambda_{j}(s)=0$ if $s>1$, let $\mathscr{C}_{j}$ denote a multitype Boolean model with cumulative intensity $\lambda_{j}$ and let $A_{j}$ be the corresponding version of the random function $A$. We may write $\lambda_{j}$ in the form at (2.5), with $f$ identically constant on $(0,1]$ and vanishing on $(1, \infty)$, and $a=D+1-\eta$ or $D+1+\eta$ according to whether $j=1$ or 2 . The process $\mathscr{C}_{1}$ may be constructed by removing sets from $\mathscr{C}$, and $\mathscr{C}_{2}$ may be constructed by adding sets of $\mathscr{C}$. If the $\mathscr{C}_{j}$ 's are derived in this way, then $A_{1} \leq A \leq A_{2}$. Applying Theorem 2.2 to the Boolean models $\mathscr{C}_{j}$ we see that the lim inf [respectively, lim sup] of $\log A(\varepsilon) /$ $\log \varepsilon$ is bounded below [above] by $2-D-\eta[2-D+\eta]$. Letting $\eta \rightarrow 0$ we deduce that $\lim \log A(\varepsilon) / \log \varepsilon$ exists and equals $2-D$, as claimed in Theorem 2.1. The case where $\lambda$ does not vanish on $(1, \infty)$ may be treated by incorporating a simple subsidiary argument; large-radius sets have negligible impact on the value of $A(\varepsilon)$.

Acknowledgment. Helpful comments by a referee have led to this more succinct version of the paper.

\section{REFERENCES}

Barnsley, M. F. (1988). Fractals Everywhere. Academic Press, London.

BERRY, M. V. and HANNAY, J. H. (1978). Topography of random surfaces. Nature 273573.

Bingham, N. H., Goldie, C. M. and Teugels, J. L. (1987). Regular Variation. Cambridge Univ. Press, New York.

Carter, P. H., CAwley, R. and Mauldin, R. D. (1988). Mathematics of dimension measurements of graphs of functions. In Proceedings of the Symposium on Fractal Aspects of Materials, Disordered Systems (D. A. Weitz, L. M. Sander and B. B. Mandelbrot, eds.) 183-186. Materials Research Society, Pittsburgh, PA.

Cutler, C. D. (1991). Some results on the behaviour and estimation of fractal dimensions of distributions on attractors. J. Statist. Phys. 62 651-708.

Dubuc, B., Zucker, S. W., Tricot, C., Quiniou, J. F. and WehBi, D. (1989). Evaluating the fractal dimension of surfaces. Proc. Roy. Soc. London Ser. A 25 113-127.

FALCONER, K. (1990). Fractal Geometry: Mathematical Foundations and Applications. Wiley, Chichester.

Hall, P. (1988). Introduction to the Theory of Coverage Processes. Wiley, New York.

Hall, P. and PolzeHL, J. (1994). On fractal-like properties of Boolean models. Research Report SRR012-94, Centre for Mathematics and its Applications, Australian National Univ.

HeRmann, H. and OHSER, J. (1993). Determination of microstructural parameters of random spatial surface fractals by measuring chord length distributions. J. Microscopy 170 87-93.

HUNT, F. (1990). Error analysis and convergence of capacity dimension algorithms. SIAM J. Appl. Math. 50 307-321.

Ling, F. F. (1990). Fractals, engineering surfaces and tribology. Wear 136 141-156.

Mandelbrot, B. B., Passoja, D. E. and Paullay, A. J. (1984). Fractal character of surfaces of metals. Nature 308 721-722.

OGata, Y. and Katsura, K. (1991). Maximum likelihood estimates of the fractal dimension for spatial patterns. Biometrika 78 463-474.

RIPLeY, B. D. (1981). Spatial Statistics. Wiley, New York.

SERINKO, R. J. (1994). A consistent approach to least squares estimation of correlation dimension in weak Bernoulli dynamical systems. Ann. Appl. Probab. 4 1234-1254. 
Stoyan, D., Kendall, W. S. and Mecke, J. (1987). Stochastic Geometry and Its Applications. Wiley, New York.

Sullivan, F. and Hunt, F. (1988). How to estimate capacity dimension. Nuclear Phys. B (Proc. Suppl.) 5A 125-128.

TAYlor, C. C. and TAYLOR, S. J. (1991). Estimating the dimension of a fractal. J. Roy. Statist. Soc. Ser. B 53 353-364.

Thomas, T. R. and Thomas, A. P. (1988). Fractals and engineering surface roughness. Surface Topography 1 143-152.

Centre for Mathematics AND iTS APPLICATIONS

AUSTRALIAN NATIONAL UNIVERSITY

CANBerra ACT 0200

AUSTRALIA

E-MAIL: Peter.Hall@anu.edu.au
KONRAD-ZuSE-ZENTRUM FÜR INFORMATIONSTECHNIK

HeILBRUNNER STR. 10

D-10711 BERLIN

GERMANY

E-MAIL:polzehl@zib-berlin.de 